\title{
Prevalência de constipação intestinal em estudantes da área da saúde
}

\author{
Marciele Monzon Ziani ${ }^{1}$, Antônio Adolfo Mattos de Castro², Simone Lara²
}

\begin{abstract}
RESUMO
Objetivo: Estimar a prevalência de constipação intestinal em acadêmicas, e verificar se os métodos diagnósticos - Escala de Avaliação da Constipação e Critérios de ROMA III - apresentam similaridade e especificidade para avaliar a constipação intestinal. Métodos: Acadêmicas da área da saúde responderam a um protocolo com questões sobre hábitos de vida, critérios de Roma III, constipação auto-referida e Escala de Avaliação da Constipação. Resultados: A prevalência da constipação intestinal de acordo com os critérios de ROMA III foi de 63,7\%, pela Escala de Avaliação da Constipação foi de $46,6 \%$, e pela constipação intestinal auto-referida $26,4 \%$. Dos fatores de risco investigados, verificou-se que 0 estresse e a ansiedade associados obtiveram associação significativa com a constipação intestinal. Observou-se que os métodos diagnósticos apresentaram boa similaridade para avaliar a presença de constipação intestinal. Conclusão: Encontrou-se uma prevalência relevante de constipação intestinal em estudantes e boa similaridade entre os métodos diagnósticos.
\end{abstract}

Descritores: Doença Crônica; Constipação Intestinal; Prevalência; Diagnóstico; Estudantes de Ciências da Saúde.

\section{Bowel constipation prevalence in health care students}

\begin{abstract}
Objective: To estimate the Bowel constipation prevalence in health care students, and to analyze the comparison of methods - the Constipation Assessment Scale and the ROMA III criteria - by means of similarity and specificity in this Bowel constipation sample. Methods: Allied health care undergraduate students answered a protocol containing questions related to health related quality of life, number of evacuations per day, ROMA III criteria for evacuations, auto referred constipation and Constipation Assessment Scale. Results: Bowel constipation prevalence was $63.7 \%$ according to ROMA III criteria, $46.6 \%$ according to Constipation Assessment Scale and $26.4 \%$ according to auto referred Bowel constipation. Psychological risk factors that presented significant association to Bowel constipation were the stress and anxiety. We also found that the ROMA III criteria and Constipation Assessment Scale presented an adequate similarity between methods. Conclusion: This study showed a relevant prevalence of Bowel constipation in students an adequate similarity between diagnosis methods.
\end{abstract}

Descriptors: Chronic Disease; Bowel Constipation; Prevalence; Diagnosis; Allied Health Undergraduate Students.

${ }^{1}$ Acadêmica de Fisioterapia na Universidade Federal do Pampa (UNIPAMPA), Urugaiana, RS, Brasil.

${ }^{2}$ Docente do curso de Fisioterapia na Universidade Federal do Pampa (UNIPAMPA), Urugaiana, RS, Brasil. 


\section{Introdução}

A constipação intestinal $(\mathrm{Cl})$ constitui-se de um distúrbio baseado em sintomas, definido como evacuação insatisfatória e caracterizado pelo hábito intestinal pouco frequente, dificuldade na eliminação das fezes, ou ambos ${ }^{1}$. É considerada um problema populacional devido a sua alta frequência ${ }^{2-4}$, e estima-se que a doença acometa um elevado percentual de indivíduos variando de $12 \%$ a $27 \%$ da população ${ }^{5-6}$. Neste contexto, a elevada incidência está associada à idade, sexo feminino, baixo nível socioeconômico, dieta inadequada, sedentarismo, alterações endócrinas e metabólicas, além de doenças neurológicas, distúrbios psiquiátricos e causas idiopáticas ${ }^{3,7-12}$.

A fisiopatologia da $\mathrm{Cl}$ é complexa e multifatorial ${ }^{13}$, e os sintomas frequentemente encontrados são as fezes ressecadas, poucas evacuações semanais (< 3 vezes), esforço para evacuar, sensação de evacuação incompleta e tempo excessivo para a evacuação ${ }^{14}$. A cronicidade dos sintomas, a falta de orientação terapêutica adequada e o uso abusivo de laxantes podem ter como implicações o surgimento de outras disfunções, como por exemplo, hemorróidas ${ }^{15}$. 0 diagnóstico da $\mathrm{Cl}$ pode ser feito por meio dos critérios de ROMA III ${ }^{12-13}$, bem como pela Escala de Constipação Intestinal - Constipation Assessment Scale (CAS), que também avalia a presença e a severidade da $\mathrm{Cl}^{16,13}$.

Considerando as modificações de hábitos de vida ao longo da vida acadêmica, e os possíveis fatores de risco relacionados ao desenvolvimento da $\mathrm{Cl}$ em estudantes universitárias, o estudo teve como objetivos estimar a prevalência de $\mathrm{Cl}$ em acadêmicas da área da saúde; verificar a associação entre esta disfunção e possíveis fatores de risco e identificar se os métodos diagnósticos da CAS e os Critérios de ROMA III apresentam similaridade e especificidade para avaliar a Cl na amostra estudada.

\section{Materiais e metódos}

Trata-se de um estudo transversal, quantitativo, no qual foi incluída uma amostra de conveniência, incluindo 212 acadêmicas do sexo feminino, compreendendo todas as faixas etárias, matriculadas regularmente no primeiro ao décimo semestre de cursos da área da saúde (enfermagem, farmácia e fisioterapia) da Universidade Federal do Pampa-UNIPAMPA, campus Uruguaiana, no primeiro semestre de 2013. Dentre os critérios de exclusão, estavam as acadêmicas que não se enquadraram nos aspectos citados, bem como acadêmicas que obtiveram algum grau de constrangimento em responder o questionário do estudo. Este projeto foi aprovado pelo Comitê de Ética e Pesquisa da universidade supracitada com o número 58425, na data de 14 de agosto de 2012. Inicialmente os pesquisadores explicaram a proposta às acadêmicas em sala de aula, e as interessadas em participar assinaram o termo de consentimento livre e esclarecido (TCLE).

O protocolo de coleta de dados utilizado constituiu-se de um questionário semiestruturado pelos pesquisadores, dividido em oito itens: dados pessoais, hábitos de vida, fatores ambientais, fatores psíquicos, número de evacuações por dia, critérios de Roma III, constipação auto-referida e a CAS.

A constipação auto-referida ${ }^{10}$ foi avaliada por meio da questão aberta: "Você tem intestino preso ou prisão de ventre?", com o objetivo de identificar a concordância entre a definição de $\mathrm{Cl}$ segundo os critérios de Roma III e a auto-percepção das acadêmicas quanto ao seu funcionamento intestinal.

A definição da $\mathrm{Cl}$ segundo os critérios de Roma III é baseada em seis critérios (esforço ao evacuar, fezes endurecidas ou fragmentadas, sensação de evacuação incompleta, sensação de obstrução, manobras manuais para facilitar as evacuações e menos de três evacuações por semana), sendo que a marcação de dois ou mais destes critérios nos últimos seis meses caracteriza a presença de $\mathrm{Cl}^{13,17}$.

A avaliação pela CAS identifica a presença e a intensidade da $\mathrm{Cl}$, e constitui-se de oito características: distensão abdominal, mudança na eliminação de gases, menor frequência de evacuações, perda involuntária de fezes líquidas, sensação de reto cheio ou pressão, dor no reto a evacuação, eliminação de fezes em menor quantidade e desejo, mas ausência de eliminação. Para cada uma destas características é dada uma escala de três pontos ("não tenho", "tenho um pouco" ou "tenho muito") representando 0,1 ou 2, respectivamente. Estas pontuações são somadas para classificar como 0 para "não constipado" e 16 para "constipação mais grave"13,18. Neste estudo optou-se por estratificar os valores das pontuações da CAS, a fim de mensurar a presença e a gravidade da $\mathrm{Cl}$. Desta forma, estes valores foram dispostos em estratos, sendo: "0-3 sem Cl", "4-7 Cl leve", "8-11 Cl moderada" e "12-16 Cl grave". 
Os dados foram descritos como média \pm desvio padrão ou como frequência absoluta e relativa. A análise de regressão logística para identificar a função dos métodos ROMA III e a CAS para o diagnóstico de $\mathrm{Cl}$, associado à razão de chance (95\% de intervalo de confiança) utilizada para identificar a chance da proporção de $\mathrm{Cl}$ segundo os critérios de ROMA III em relação à idade, local que realiza as refeições, número de refeições, com quem reside, ingestão de líquidos, ingestão de fibras, ingestão de frituras, pratica de atividade física, efeitos psíquicos, uso de medicações. 0 gráfico de BlandAltman foi construído para identificar a similaridade entre o método de ROMA III e a CAS. A área abaixo da curva (curva $\mathrm{ROC}$ ) foi plotada para identificar a sensibilidade e a especificidade dos métodos ROMA III e a CAS. Foi considerado como significante um $p<0,05$. Os dados foram analisados por meio do programa estatístico SPSS®, versão 17.0.

\section{Resultados}

Foram incluídas neste estudo 212 acadêmicas da área da saúde (43 acadêmicas do curso de enfermagem, 57 do curso de farmácia, 105 de curso de fisioterapia, e 07 acadêmicas não responderam o curso de origem), com idades entre 17 à 24 anos (média etária de 23,7 anos). A tabela 1 demonstra os possíveis fatores associados com a $\mathrm{Cl}$ de acordo com os critérios de ROMA III, nas constipadas. Evidenciou-se que a variável idade, a partir do cálculo da mediana ( $\leq 22$ anos e > 22 anos), não demonstrou associação significativa com esta disfunção.

Da mesma forma, os fatores relacionados com os hábitos de vida não mostraram associação com a $\mathrm{Cl}$. Dentre esses fatores, destaca-se que $35,8 \%$ das estudantes reside com os pais, $76,9 \%$ realiza suas refeições em casa, $54,7 \%$ faz quatro ou mais refeições diárias, $93,4 \%$ refere a ingestão de líquidos, $67,9 \%$ o consumo de fibras, e 59,9\% retrata não praticar nenhum tipo de atividade física regular. Com relação à análise dos fatores psíquicos, verificou-se que as variáveis de estresse e ansiedade associadas, e somente o estresse, demonstraram associação significativa com a $\mathrm{Cl}(p<0,01)$.

Tabela 1 - Caracterização da amostra através dos possíveis fatores associados com a Cl de acordo com os critérios de ROMA III

\begin{tabular}{|c|c|c|}
\hline Variáveis & $\begin{array}{c}\text { Constipadas } \\
\text { (ROMA III) } \\
\text { n (\%) }\end{array}$ & $\mathrm{p}$ \\
\hline Idade $\leq 22$ years & $67(31)$ & 0.8 \\
\hline Idade $>22$ years & $67(31)$ & \\
\hline Reside com os pais & $45(21)$ & 0.2 \\
\hline Não reside com os pais & $90(43)$ & \\
\hline Realiza refeições em casa & $100(53)$ & 0.07 \\
\hline $\begin{array}{c}\text { Realiza as refeições em lanchonetes e } \\
\text { restaurantes }\end{array}$ & $20(11)$ & \\
\hline Quatro ou mais refeições diárias & $73(35)$ & 1.0 \\
\hline Menos que quarto refeições diárias & $61(29)$ & \\
\hline Ingesta de líquidos & $127(60)$ & 0.5 \\
\hline Ingesta de fibras & $91(43)$ & 0.7 \\
\hline Ingesta de frituras & $29(14)$ & 0.7 \\
\hline Sedentarismo & $86(41)$ & 0.1 \\
\hline Depressão & $5(2)$ & 1.0 \\
\hline Estresse & $44(15)$ & 0.0001 \\
\hline Ansiedade & $59(28)$ & 0.14 \\
\hline Estresse e ansiedade & $30(20)$ & 0.01 \\
\hline Uso de medicação & $17(8)$ & 0.5 \\
\hline
\end{tabular}

Dentre os critérios de Roma III avaliados, a necessidade de esforço para evacuar e a presença de fezes endurecidas, aparecem como mais prevalentes, $46,7 \%$ e 50\% respectivamente, quando comparadas aos demais. Outro aspecto relevante sobre os critérios de ROMA III foi à frequência predominante de evacuações semanais de até quatro vezes, encontrada em $66 \%$ das estudantes. 
Com relação aos critérios diagnósticos, apenas $26,4 \%$ das estudantes referiram sintomas de $\mathrm{Cl}$ em algum momento da vida, através da questão sobre a $\mathrm{Cl}$ auto-referida. Por outro lado, a prevalência de $\mathrm{Cl}$ a partir dos critérios de ROMA III foi de $63,7 \%$, havendo uma diferença estatística importante entre os dois métodos $(p<0,01)$.

A fim de apresentar os dados do método de avaliação da CI pela CAS, as pontuações foram dispostas por estratos, sendo $53,3 \%$ sem Cl, 34,6\% Cl leve, 9,6\% Cl moderada e 2,4\% Cl grave, e 46,6\% se considerarmos a presença de $\mathrm{Cl}$ em qualquer grau. Este dado vai ao encontro dos achados pelos critérios de ROMA III, na qual a presença de $\mathrm{Cl}$ também foi relevante.

A figura 1 (Bland Altman) demonstrou que os métodos de ROMAIII e CAS apresentaram uma similaridade entre si para avaliar a presença de $\mathrm{Cl}$. Este dado pode estar relacionado ao fato que ambos se utilizam de sintomas semelhantes para a verificação da Cl13. Na figura 2 (Curva ROC), no qual são comparados os métodos diagnósticos, verificou-se um bom poder de triagem para indivíduos constipados, observando-se uma especificidade de 60\% entre eles.

Figura 1- Bland Altman para ROMA III e CAS

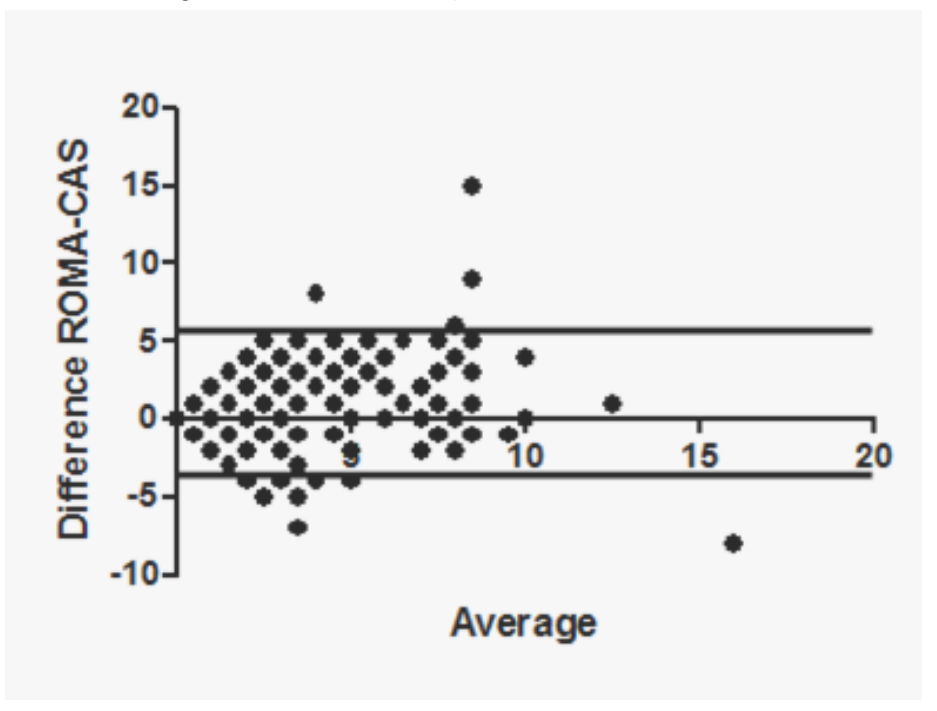

Figura 2 - Curva ROC para Cl pelo ROMA III e CAS

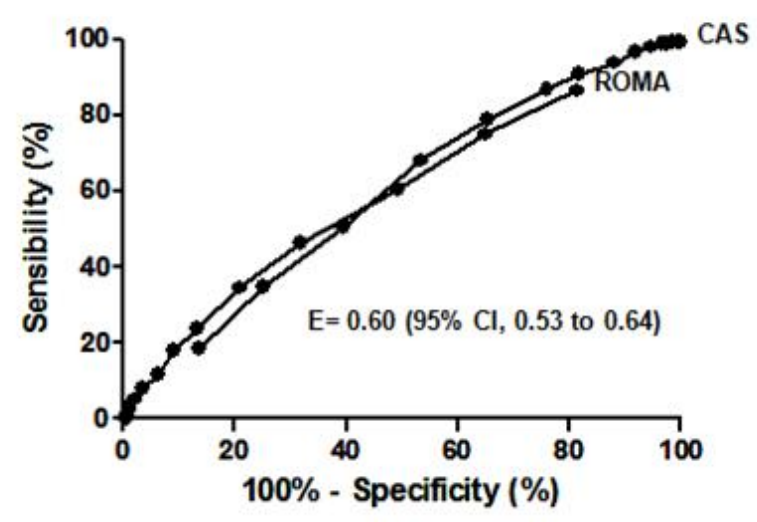

\section{Discussão}

Considerando os fatores associados com a $\mathrm{Cl}$, evidenciou-se que a variável idade, não demonstrou associação significativa com esta disfunção. Por outro lado, um estudo semelhante encontrou que a taxa de $\mathrm{Cl}$ reduziu com 0 avanço da idade (42,1\% e 10,5\% na segunda e terceira década de vida, respectivamente) $)^{19}$. O mesmo autor afirma que as manifestações da $\mathrm{Cl}$ têm início na adolescência, podendo se estender até os 21 anos, já que é nesse período que se iniciam as modificações hormonais e comportamentais ${ }^{19}$. 
Com relação aos hábitos de vida, grande parte das estudantes relatou residir com os pais, realizar suas refeições em casa, e fazer quatro ou mais refeições diárias, não havendo associação significativa entre esses e o desenvolvimento de $\mathrm{Cl}$. Apesar destes achados, destaca-se que as responsabilidades da vida acadêmica costumam acarretar uma série de modificações na vida destas jovens, em especial, alterações em seus hábitos alimentares, interferindo assim em sua qualidade de vida e constituindo-se um fator de risco para a $\mathrm{Cl}^{20}$.

De fato, não realizar refeições em horários regulares, e nem fracioná-las pode implicar em diminuição de reflexos gastrocólicos pelo volume inadequado de alimento para formação do bolo fecal, bem como à falta de estímulo do movimento de peristaltismo, e assim, levar ao quadro de $\mathrm{Cl}^{15,21}$. Alguns trabalhos encontraram uma prevalência de constipação de $51,9 \%, 55 \%$ e $66,6 \%$, respectivamente, em indivíduos que realizavam quatro ou mais refeições, fato este que evidencia a associação entre um maior número de refeições e o desenvolvimento da $\mathrm{Cl}^{22-24}$, dados estes que vão de encontro aos do atual estudo.

Os dados do presente estudo corroboram os de outros trabalhos, ao evidenciar que nem a ingestão de fibra alimentar, e nem de líquidos estão associadas com a $\mathrm{Cl}^{10,25}$. Por outro lado, autores ressaltam que uma dieta pobre em fibras alimentares contribui para o desenvolvimento da $\mathrm{Cl}^{4,10}$, e existe um efeito laxante da fibra alimentar na produção aumentada de fezes e no tempo acelerado no trânsito do cólon11, auxiliando no funcionamento intestinal adequado.

Da mesma forma, este hábito pode estar associado com o meio acadêmico, uma vez que devido à intensa carga horária, as estudantes podem omitir ou substituir suas refeições por lanches rápidos e práticos, pobres em fibras ${ }^{23}$. Porém uma explicação plausível para a prevalência razoável de ingestão de fibras encontrada neste estudo, é que, por serem estudantes da área da saúde, imagina-se que tenham uma alimentação mais saudável e equilibrada.

Avida sedentária, comum na atualidade e prevalentenos dados do atual estudo, ainda que fator de risco para o desenvolvimento da $\mathrm{Cl}$, não teve relação estatística neste trabalho. Achados semelhantes detectaram que $44,4 \%$ dos indivíduos constipados não praticavam exercícios físicos, ao passo que no grupo dos não constipados, foi de $70,9 \%^{23-24}$. Contudo, outro estudo em jovens universitários encontrou prevalência de sedentarismo em $65 \%$ dos constipados e $60,7 \%$ dos não constipados 22 .

Sob esta perspectiva, autores retratam uma série de efeitos fisiológicos positivos do exercício físico sobre o sistema gastrointestinal, como por exemplo, o estímulo ao peristaltismo, melhora do tônus muscular da musculatura pélvica e abdominal e a melhora do padrão intestinal ${ }^{26-27}$. Por outro lado, há relatos que tanto a ingestão de líquidos, quanto a prática de atividade física não influenciam na $\mathrm{Cl}$, nem promovem o funcionamento intestinal regular ${ }^{9}$. Em conformidade com tais achados, autores não encontraram diferenças significativas dos níveis de atividade física entre indivíduos constipados e não constipados ${ }^{28}$.

De forma semelhante aos dados do presente estudo, no que refere aos fatores psíquicos, achados reportam uma associação entre a $\mathrm{Cl}$ e a ansiedade ${ }^{19}$. Ainda, um importante fator a ser considerado no que tange ao desenvolvimento da $\mathrm{Cl}$ é que as condições psicológicas parecem predispor a esta disfunção, com possível implicação na patogênese da mesma ${ }^{2}$. Verifica-se, que estes sintomas podem estar presentes nas universitárias, pelas circunstâncias, como a escolha da profissão, o enfrentamento das responsabilidades da vida acadêmica, a distância da família, podendo apresentar maior suscetibilidade ao desenvolvimento de fatores psíquicos. É importante ressaltar que, apesar das demais variáveis analisadas não demonstrarem associação significativa com a $\mathrm{Cl}$ segundo os critérios de ROMAIII, devem ser considerados como potenciais fatores de risco para o desenvolvimento da $\mathrm{Cl}$.

Uma explicação plausível para a discrepância de resultados encontrados entre os métodos diagnósticos da $\mathrm{Cl}$ autoreferida e critérios de ROMA III, utilizados no presente estudo, pode estar associada ao fato de que a $\mathrm{Cl}$ é considerada pelos indivíduos apenas uma diminuição da frequência evacuatória. Entretanto, sabe-se que esta síndrome se manifesta através de uma variedade de sintomas que variam não apenas em complexidade, mas também em cronicidade e intensidade, fato este não percebido pelas estudantes. Além disto, esta diferença encontrada entre os métodos pode estar associada ao fato de que o auto-relato, por ser um critério subjetivo e influenciado por costumes sociais, tem acurácia inferior quando comparada aos critérios baseados em sintomas ${ }^{3}$.

Assim, os critérios de ROMA III foram desenvolvidos para padronizar a definição de constipação em estudos epidemiológicos, e para realizar o diagnóstico na prática clínica ${ }^{1}$. Contudo em outro estudo, verificou-se história relatada de sintomas de $\mathrm{Cl}$ em $72 \%$ das acadêmicas, e mediante o preenchimento dos critérios de Roma III em $55 \%$ das mulheres estudadas ${ }^{2}$. Porém, outros trabalhos encontraram uma prevalência de $\mathrm{Cl}$ de $64 \%$ e $72,5 \%$ de acordo com os critérios de ROMA II e ROMA III respectivamente, em estudantes do sexo feminino ${ }^{22,29}$; prevalências semelhantes aos do atual estudo. Alguns estudos demonstraram que a $\mathrm{Cl}$ manifesta-se mais frequentemente em mulheres jovens, solteiras e sem qualquer motivo para alterações anatômicas ou fisiológicas do assoalho pélvico ${ }^{22,29}$, sendo essas características compatíveis com grande parte das acadêmicas. 
Corroborando, este contexto pode estar relacionado com questões culturais e comportamentais, conforme afirma Santos9, que explica que desde a infância, a menina é orientada a não utilizar o vaso sanitário em lugares públicos. Isto se intensifica a partir da ampliação das atividades cotidianas, estudantis e laborais. Nesse conjunto, as necessidades fisiológicas que requerem um ambiente mais íntimo acabam sendo deixadas em favor das atividades prioritárias naquele momento, e este adiamento das evacuações por alguns dias torna a evacuação não prazerosa. Assim, os fatores comportamentais associados às refeições rápidas e pobres em fibras, ao estilo sedentário de vida e à postura de controle intestinal, tornam-se fatores agravantes para o desenvolvimento da $\mathrm{Cl}^{9}$.

Os critérios de Roma III mais prevalentes, referidos pelas acadêmicas do estudo, foram a necessidade de esforço para evacuar e a presença de fezes endurecidas. De fato, um estudo encontrou dados semelhantes, de $48 \%$ e $52 \%$ em relação ao esforço evacuatório e ao aspecto endurecido das fezes, respectivamente ${ }^{2}$, apesar de outros trabalhos reportarem a prevalência de outros sintomas gastrintestinais, como sensação de evacuação incompleta ${ }^{15,29}$. Adicionalmente, outro fator relevante sobre os critérios de ROMA III encontrado no estudo foi à frequência predominante de evacuações semanais de até quatro vezes pelas acadêmicas. Há achados que referem que a maioria das constipadas apresentou uma frequência de evacuações inferior a três vezes semanais ${ }^{15,29}$.

Por meio das análises realizadas no atual estudo, evidenciou-se um bom nível de similaridade e especificidade entre os métodos diagnósticos CAS e ROMA III. Sendo assim, pode-se inferir que os métodos apresentaram uma similaridade entre si para avaliar a presença de $\mathrm{Cl}$, bem como são capazes de excluir corretamente aqueles que não possuem $\mathrm{Cl}$, ou seja, destaca o quão específicos são os métodos utilizados no presente estudo.

\section{Considerações Finais}

Percebeu-se uma alta prevalência de $\mathrm{CI}$ na amostra avaliada, de acordo com os critérios de Roma III, apesar de ser menos frequente quando auto-referida pelas acadêmicas, demonstrando um déficit de conhecimento das mesmas acerca desta disfunção. Verificou-se uma associação desta síndrome com sintomas psíquicos, resultados estes esperados, tendo em vista as atuais condições na qual pertence à amostra estudada. Observou-se que os métodos diagnósticos utilizados apresentaram uma boa similaridade e especificidade para avaliar a presença de $\mathrm{Cl}$ na amostra, demonstrando que ambos podem ser utilizados com confiabilidade para diagnosticar esta disfunção.

Por fim, um conhecimento mais aprofundado sobre questões associadas ao desenvolvimento da $\mathrm{Cl}$, bem como seus métodos diagnósticos, foram algumas das contribuições do estudo. Ainda, devido à escassez de estudos na área, acreditase que outros trabalhos devam ser desenvolvidos, uma vez que, questões comportamentais, podem sinalizar risco para 0 desenvolvimento de $\mathrm{Cl}$. É necessário também fomentar ações baseadas na educação em saúde, no sentido de promover um maior nível de conhecimento sobre esta disfunção, possa auxiliar estas acadêmicas a prevenir o aparecimento de possíveis doenças intestinais em fases mais tardias.

\section{Referências Bibliográficas}

1. Locke GR, Pemberton JH, Phillips SF. AGA technical review on constipation. American Gastroenterological Association. Gastroenterol. 2000; 119(6):1766-78.

2. Trisóglio C, Marchi CM, Torres US, Netinho JG. Prevalência de Constipação Intestinal entre Estudantes de Medicina de uma Instituição no Noroeste Paulista. Rev bras Coloproct. 2010; 30(2): 203-209.

3. Talley NJ. Definitions, epidemiology, and impact of chronic constipation. Rev Gastroenterol Disord. 2004; 4 Supl 2:3-10.

4. Arnaud MJ. Mild dehydration: a risk factor of constipation? European Journal of Clinical Nutrition. 2003; 57 Supl 2:88-95.

5. Machado WM, Capelari SM. Avaliação da eficácia e do grau de adesão ao uso prolongado de fibra dietética no tratamento da constipação intestinal funcional. Rev. Nutr. 2010; 23(2): 231-238.

6. Cheng CW, Bian ZX, Zhu LX, Wu JC, Sung JJ. Efficacy of a Chinese Herbal Proprietary Medicine (Hemp Seed Pill) for Functional Constipation. Am J Gastroenterol. 2011; 106(1):120-129.

7. César MAP, Uemura LA, Passos MPS, Bassi DG, Paula PR de. Colectomias no Tratamento Cirúrgico da Constipação Intestinal Crônica - Relato de Quatro Casos. Rev bras Coloproct. 2008; 28(2): 241-245. 
8. Da Cunha GH, De Moraes MEA, De Oliveira JC. Condutas terapêuticas no manejo da constipação crônica. Revista Eletrônica Pesquisa Médica. 2008; 2(4):11-17.

9. Santos JJCM. Opiniões e revisão bibliográfica- Constipação Intestinal. Revista Brasileira Coloproct. 2005; 25(1):79-93. 10. Murakami K, Okubo H, Sasaki S. Dietary intake in relation to self-reported constipation among Japanese women aged 18-20 years. European Journal of Clinical Nutrition. 2006; 60(5): 650-657.

11. Hongisto SM, Paajanen L, Saxelin M, Korpela R. A combination of fibre-rich rye bread and yoghurt containing Lactobacillus GG improves bowel function in women with self-reported constipation. European Journal of Clinical Nutrition. 2006; 60(3): 319-324.

12. Gallegos-Orozco JF, Foxx-Orenstein AE, Sterler SM, Stoa JM. Chronic Constipation in the Elderly. Am J Gastroenterol. 2012; 107:18-25.

13. Consenso Brasileiro de Constipação Intestinal Induzida por Opioides 2009. Revista brasileira de cuidados paliativosSaúde \& Qualidade de Vida - Patologia \& Nutrição. Disponível em:<http://www.rgnutri.com.br/sqv/patologias/cbc.php>. Acesso em: 13 outubro 2012.

14. Vieira EP, Pupo Neto JA, Lacombe DLP. Contribuição da Manometria Ano Retal na Avaliação da Constipação Intestinal Crônica. Rev bras Coloproct. 2005; 25(0): 348-360.

15. Collete VL, Araújo CL, Madruga SW. Prevalência e fatores associados à constipação intestinal: um estudo de base populacional em Pelotas, Rio Grande do Sul, Brasil, 2007. Cad. Saúde Pública. 2010; 26(7): 1391-1402.

16. McCrea GL, Miaskowski C, Stotts NA, Macera L, Hart SA, Varma MG. Review Article: Self-Report Measures to Evaluate Constipation. Aliment Pharmacol Ther. 2008; 27(8): 638-648.

17. Drossman DA. The functional gastrointestinal disorders and the Rome III process. Gastroenterology. 2006; 130(5):1377-90. 18. Leão EP, Pena CJM, Araújo SM, Gomes ML. Physical therapy combined with a laxative fruit drink for treatment of chagasic megacolon. Arq. Gastroenterol. 2011; 48(1): 52-57.

19. Silva DO, Da Silva FI, Machado DZ, Passoni CRMS. Prevalência e correlação entre constipação intestinal e ansiedade. Cadernos da Escola de Saúde. 2012; 6:70-83.

20. Billodre BN; Gomez R; Caletti G. Prevalência e motivação de uso de laxantes entre universitárias do curso de nutrição do Centro Universitário Metodista de Porto Alegre/RS. Ciência em Movimento - Biociências e Saúde. 2013; 15(30): 35-43. 21. Magalhães ACP, Chaud, DMA, Marchioni DML, Souza LK, Yamanaka SM. Prevalência e fatores de risco para constipação intestinal em estudantes universitários. Nutr Pauta. 2002; (56):13-7.

22. Cota RP, Miranda LS. Associação entre constipação intestinal e estilo de vida em estudantes universitários. Rev Bras Nutr Clin. 2006; 21(4):296-301.

23. Jaime RP, Campos RC, Santos TST, Marques MS. Prevalência e fatores de risco da constipação intestinal em universitários de uma instituição particular de Goiânia, GO. Rev Inst Ciênc Saúde. 2009; 27(4):378-83.

24. Braz CR, Lessa NMV. Fatores de risco e prevalência de constipação intestinal em graduandos do curso de nutrição de um centro universitário de Minas Gerais. Nutrir Gerais. 2011; 5(8): 740-754.

25. Murakami K, Sasaki S, Okubo H, Takahashi Y, Hosoi Y, Itabashi M. Association between dietary fiber, water and magnesium intake and functional constipation among young Japanese women. European Journal of Clinical Nutrition. 2007; 61(5): 616-622.

26. Dukas L, Walter CW, Giovannucci EL. Association between physical activity, fiber intake, and other lifestyle variables and constipation in study of women. Am J Gastroenterol. 2003; 98(8): 1790-6.

27. Simren, M. Physical activity and the gastrointestinal tract. European Journal of Gastroenterology \& Hepatology. 2002; 14(10): 1053-1056.

28. Tuteja AK, Talley NJ, Joos SK, Woehl JV, Hickam DH. Is constipation associated with decreased physical activity in normally active subjects? Am J Gastroenterol. 2005; 100(1):124-9.

29. Martinoff T, De Aquino RC. Avaliação de constipação intestinal e sua relação com hábito alimentar e estilo de vida de universitários. Revista Brasileira de Ciências da Saúde. 2008; 3(15): 47-51. 


\section{Marciele Monzon Ziani}

Endereço para correspondência - Universidade Federal do Pampa (UNIPAMPA); Faculdade de Ciências da Saúde, Campus Uruguaiana, BR 472, km 592, CP 118, CEP 97500-970, Cidade: Uruguaiana, RS, Brasil.

E-mail: marcieliziane@gmail.com

Lattes: http://lattes.cnpq.br/1674451108446102

Antônio Adolfo Mattos de Castro - antonioamcastro@yahoo.com.br

Simone Lara - simonelara@unipampa.edu.br

Enviado em 04 de setembro de 2014. Aceito em 29 de abril de 2015. 